\title{
Cisplatin-loaded, Sialyl Lewis X-Modified Liposomes: Drug Release, Biodistribution and Antitumor Efficacy
}

\author{
SHUICHI KISHIMOTO, NOBUHITO FUJITANI, TAKANORI OHNISHI, \\ HIROKI AOKI, RYOSUKE SUZUKI and SHOJI FUKUSHIMA
}

Department of Pharmaceutics, Faculty of Pharmaceutical Sciences, Kobe Gakuin University, Kobe, Japan

\begin{abstract}
Background/Aim: The aim of this study was to evaluate the characteristics of two cisplatin (CDDP)-loaded, sialyl Lewis X-modified liposomes (CDDP-SLX-LIP) with different particle size. Each had the same lipid composition and CDDP concentration, but a different drug-to-lipid ratio. Materials and Methods: The amount of platinum in the filtrates was determined by the ICP method. The antitumor effect was assessed using rats bearing MRMT-1 tumor. Results: CDDP release was faster from the larger liposome formulation (L-LIP) than from the smaller one (S-LIP). Treatment with the two CDDP-SLX-LIPs resulted in comparable antitumor effects without obvious side-effects, but treatment with CDDP solution resulted in significant weight loss and an elevated BUN. Tumor accumulation of CDDP-SLX-LIP was clearly greater than that of CDDP solution and accumulation of S-LIP was greater than that of L-LIP. Conclusion: CDDP-SLX-LIP exhibited antitumor effects depending on their release behavior and tumor accumulation.
\end{abstract}

Cisplatin (CDDP) has a strong antitumor activity and is routinely used as a first-line treatment of testicular, ovarian, cervical, head and neck, bladder, small-cell lung, and nonsmall-cell lung cancers. Nephrotoxicity and ototoxicity are two side-effects that can be cumulative and irreversible, potentially limiting CDDP treatment. In patients with impaired renal function, the administration of CDDP is avoided. Platinum derivatives have less renal toxicity, but do not have better antitumor characteristics than does CDDP (1).

Correspondence to: Department of Pharmaceutics, Faculty of Pharmaceutical Sciences, Kobe Gakuin University, Minatojima 11-3, Chuo-ku, Kobe 650-8586, Japan. Tel: +81 789744753, Fax: +81 789744753, e-mail: skisimot@pharm.kobegakuin.ac.jp

Key Words: Cisplatin, liposome, sialyl Lewis $\mathrm{X}$, release, accumulation.
There have been numerous reports of the tumor-targeting delivery of antitumor agents using liposomes. Among them, liposomes that incorporate doxorubicin have often been used $(2,3)$. Entrapment of liposomes in the reticuloendothelial system (RES) has been a major barrier for tumor-selective delivery. Polyethylene glycol (PEG) modification of liposomes enables liposomes to avoid entrapment in the RES and these PEG-coated liposomes have demonstrated prolonged blood circulation time and increased accumulation in tumors $(4,5)$. Therefore, Doxil ${ }^{\circledR}$, a PEG-coated liposome containing doxorubicin, is currently on the market. Liposome formulations that incorporate CDDP, such as SPI-077 and Lipoplatin, have also been evaluated for reduced renal toxicity and improved tumor- selectivity (6-9). SPI-077 is a formulation that encapsulated CDDP in stealth liposomes composed of hydrogenated soy phosphatidylcholine (HSPC), cholesterol $(\mathrm{CH})$, and PEG-modified distearyl phosphatidylethanolamine (DSPE) and was similar in composition to Doxil ${ }^{\circledR}(10,11)$. Despite its superior pharmacokinetic properties, SPI-077 did not progress to Phase III trials because of a lack of activity in Phase II trials. Lipoplatin is a liposomal CDDP prepared using HSPC, $\mathrm{CH}$, dipalmitoyl phosphatidylglycerol (DPPG), and methoxyPEG-DSPE $(12,13)$. It was tested in a number of malignancies in several Phase II and III trials, including those for pancreatic cancer, head and neck cancer, breast cancer, and gastric cancer. Those clinical trials have shown Lipoplatin to produce similar efficacy to that of CDDP with significant toxicity reduction. In addition, amphiphilic block copolymer micelles are well-suited for solubilization of hydrophobic drugs. Incorporation of CDDP into such copolymers proceeds through the formation of coordination bonds between CDDP and the polyion block of the block copolymer, which also induces micelle formation $(8,9)$. Complexation of CDDP with PEG-poly (aspartic acid) copolymers led to the spontaneous formation of stable polymer micelles with high drug loading. Preclinical studies demonstrated increased circulation of the micelle in plasma and increased drug exposure of the tumors $(14,15)$. 
Sialyl Lewis X (SLX) is a molecule that specifically and efficiently targets E-selectin. Therefore, SLX-coated liposomes displayed a high affinity for E-selectin expressed on vascular endothelial cells and enhanced accumulation in tumors and inflammatory tissue without PEG modification (16-18). Hirai et al. (19) developed a novel efficient technology to encapsulate CDDP in liposomes. The character of cis-diamminedinitratoplatinum (II), which is highly water soluble and readily converted to CDDP in the presence of chloride ions, was exploited to encapsulate CDDP in liposomes conjugated with SLX at high concentrations. The SLX-coated liposomes encapsulating CDDP (CDDP-SLXLIP) were expected to enhance the pharmaceutical availability of CDDP and the selectivity of the antitumor effect. We have two formulations of CDDP-SLX-LIP; a smaller liposome formulation (S-LIP) and a larger liposome formulation (L-LIP), each with different average diameters, but the same lipid composition. In this study, we examined the release profiles of CDDP from the CDDP-SLX-LIPs, the toxicity to the liver and kidneys, the in vivo antitumor effects on rat MRMT-1 mammary tumor cells, and the tissue and tumor distribution of CDDP.

\section{Materials and Methods}

Cells. Rat MRMT-1 mammary tumor cells were kindly supplied by the Cell Resource Center for Biomedical Research, Tohoku University. Cells were maintained in minimum essential medium containing $10 \%$ fetal bovine serum at $37^{\circ} \mathrm{C}$ in humidified air with $5 \% \mathrm{CO}_{2}$.

Animals. Female Sprague-Dawley (SD) rats, 4 to 5 weeks old, weighting 150 to $180 \mathrm{~g}$, were purchased from Charles River Laboratories Japan Inc. (Yokohama, Japan). The animals were housed with free access to food and water at $25^{\circ} \mathrm{C}$ with a $12-\mathrm{h}$ alternating light/dark cycle.

Liposomes. All liposomes were supplied by Katayama Chemical Industries Co., Ltd. (Osaka, Japan). Liposomes were prepared using the method described by Hirai et al. (19) with an improved dialysis method.

$C D D P$ release from $C D D P-S L X-L I P$. CDDP-SLX-LIP was added to saline or saline containing $5 \%$ albumin, then incubated at $37^{\circ} \mathrm{C}$. At the indicated times $(1,3,5$ and 7 days), the mixture was filtered using a Vivaspin 500 protein concentrator (Sartorius, Tokyo, Japan) with a $300 \mathrm{kDa}$ molecular weight cutoff value to exclude liposomes. The amount of platinum in the filtrates was determined using an SPS 3100 ICP Optical Emission Spectrometer (Hitachi High-Tech Science Corporation, Tokyo, Japan).

Antitumor activity. SD rats were inoculated in the left flank with $2 \times 10^{5}$ MRMT- 1 cells. When tumors grew to approximately $8 \mathrm{~mm}$ in diameter, drug was administered via the tail vein. The first day in which the drugs were administered was considered day 0 . As such, the drugs were administered on day 0 , day 5 , and day 10 . The cumulative dose of CDDP solution was $12 \mathrm{mg} / \mathrm{kg}$, and that of
CDDP-SLX-LIP was $24 \mathrm{mg} / \mathrm{kg}$. The lipid dose of SLX-LIP (control liposome without CDDP) was equal to that of CDDP-SLX-LIP at a dose of $24 \mathrm{mg} / \mathrm{kg}$. Rats were weighed and tumors were measured twice a week. The tumor volume was measured once 2 or 3 days in two dimensions using a caliper, and tumor volume was calculated using the following equation:

Volume $\left(\mathrm{mm}^{3}\right)=$ longer diameter $\times(\text { shorter diameter })^{2} \times 0.5$

Toxicity study. Normal SD rats were administered CDDP solution or CDDP-SLX-LIP via the tail vein. The dose of CDDP solution was $4 \mathrm{mg} / \mathrm{kg}$, and that of CDDP-SLX-LIP was $8 \mathrm{mg} / \mathrm{kg}$. Blood was collected via the cervical vein just before and 4-days after drug administration. The samples were sent out to Oriental Yeast Co., Ltd. (Shiga, Japan), where the levels of serum ALT and BUN were measured.

Tissue distribution. SD rats were inoculated in the left flank with $2 \times 10^{5}$ MRMT- 1 cells. When the tumors grew to approximately 12 $\mathrm{mm}$ in diameter, the drug administration was carried out via the tail vein for each 20 rats. The dose of free CDDP was $4 \mathrm{mg} / \mathrm{kg}$, and that of CDDP-SLX-LIP was $8 \mathrm{mg} / \mathrm{kg}$. At the indicated times $(1,6,24$, and $48 \mathrm{~h}$ ), blood was collected via the cervical vein and tumor tissue, lung, liver, spleen, and kidney were removed from 5 rats at each time for each drug and washed with saline. The plasma and tissues were homogenized with a mixture of perchloric acid and hydrogen peroxide solution $(1: 2)$ and digested at $60^{\circ} \mathrm{C}$ for $48 \mathrm{~h}$. The amount of platinum in the samples was determined using an SPS 3100 ICP Optical Emission Spectrometer (Hitachi High-Tech Science Corporation, Tokyo, Japan).

Statistical analysis. Results are expressed as mean \pm standard error of the mean. Statistical analysis was performed using Student's $t$ test or Dunnett's test for the single or multiple comparisons of experimental groups, respectively, using Microsoft Excel. A value of $p<0.05$ was considered significant.

\section{Results}

Characteristics of CDDP-SLX-LIP. Two types of CDDP-SLXLIP were assessed. The liposomes were differentiated by size; the small sized CDDP-SLX-LIP (S-LIP) was obtained by filtering the large sized CDDP-SLX-LIP (L-LIP) using a Thermobarrel extruder. The mean diameter of S-LIP and L-LIP was $131 \mathrm{~nm}$ and $182 \mathrm{~nm}$, respectively. The liposome had the same lipid composition, containing dipalmitoylphosphatidylcholine (DPPC), cholesterol, ganglioside GM3, dihexadecyl phosphate, and dipalmitoylphosphatidylethanolamine (DPPE) (molar ratio 32:36.5:7:17.5:7, respectively); therefore, they displayed a similar zeta-potential $(-51 \mathrm{mV}$ and $-53 \mathrm{mV}$ for S-LIP and L-LIP, respectively). To adjust the CDDP concentration to $2 \mathrm{mg} / \mathrm{ml}$, L-LIP contained a lipid concentration of $28.7 \mathrm{mg} / \mathrm{ml}$. However, S-LIP required a higher lipid concentration of $60.1 \mathrm{mg} / \mathrm{ml}$ to maintain the same CDDP concentration. Because of the difference in internal space between the two liposomes, S-LIP required more liposome particles to contain the same amount of CDDP as L-LIP. 
Release from CDDP-SLX-LIP. The release of CDDP from the liposome formulations into saline was assessed by determining the amount of platinum in filtrates after ultrafiltration of saline containing liposomes. As shown in Figure 1, both liposome formulations released platinum compounds into saline immediately after immersion, and the initial release of L-LIP (37\%) was much higher than that of S-LIP (7.9\%). Thereafter, the release profile of S-LIP was also slower than that of L-LIP. After 7 days, the release rates of L-LIP and S-LIP were 55\% and 18\%, respectively.

Antitumor effects. Drugs were administered 3 times intravenously via the tail vein. Three times injections of CDDP solution at $4 \mathrm{mg} / \mathrm{kg}$ was tolerated, whereas some rats administered $6 \mathrm{mg} / \mathrm{kg}$ were found dead after the second or third injection (data not shown). CDDP solution significantly inhibited the growth of MRMT-1 tumors and suppressed weight gain in rats (Figures 2 and 3). Both CDDP-SLX-LIPs significantly inhibited tumor growth without suppressing weight gain. There was no difference in tumor growth inhibition between S-LIP and L-LIP. Furthermore, the antitumor effects of CDDP-SLX-LIP (3 doses of $8 \mathrm{mg} / \mathrm{kg}$ ) were comparable to those of CDDP solution (3 doses of 4 $\mathrm{mg} / \mathrm{kg}$ ). However, there were obvious differences in hair loss and suppression of weight gain between rats administered CDDP-SLX-LIP and those administered CDDP solution. Hair loss was observed in all rats administered CDDP solution, but there was no hair loss in the group administered CDDP-SLX-LIP.

Side-effects. Side-effects were assessed using normal SD rats that received a single injection of CDDP solution $(4 \mathrm{mg} / \mathrm{kg})$ or S-LIP $(8 \mathrm{mg} / \mathrm{kg})$ via the tail vein. BUN and ALT levels were compared between before and 4-days after administration (Figure 4). Administration of CDDP solution resulted in significantly increased BUN levels 4 days after administration, but S-LIP did not affect BUN levels. Conversely, ALT levels were not affected in either treatment group.

Biodistribution. Platinum levels in plasma and tissues were measured 1, 24, and $48 \mathrm{~h}$ after administration of CDDP solution $(4 \mathrm{mg} / \mathrm{kg})$ or CDDP-SLX-LIP $(8 \mathrm{mg} / \mathrm{kg})$ via the tail vein in normal SD rats (Figure 5). Both types of CDDPSLX-LIP were retained longer in blood than CDDP solution and administration of S-LIP resulted in several times higher platinum levels in plasma than administration of L-LIP at 1 and $24 \mathrm{~h}$. In the kidney, there was no difference in platinum levels among the 3 groups. In the spleen, lung, and liver, there was a clear difference in platinum levels between CDDP solution and CDDP-SLX-LIP. Administration of SLIP resulted in higher platinum levels in lung and liver tissue than administration of L-LIP, but there was no difference in

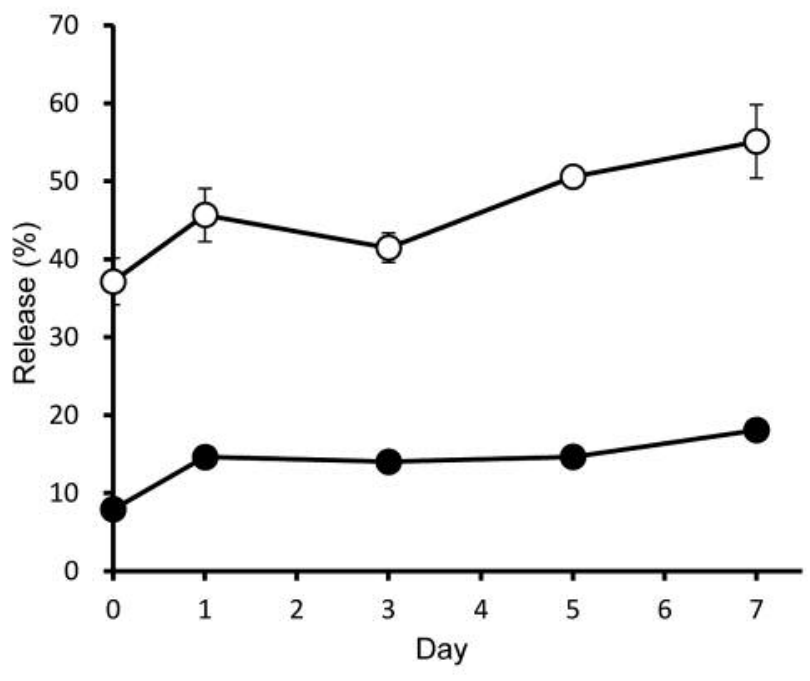

Figure 1. In vitro drug release profiles of CDDP-SLX-LIP in saline at $37^{\circ} \mathrm{C}$. Liposomes were incubated in saline. Platinum amounts released in saline were measured by ICP-AES. Open symbol: L-LIP; closed symbol: S-LIP. Data represent the mean $\pm \operatorname{SEM}(n=5)$.

platinum levels in the spleen tissue of both groups. In tumor tissue, the platinum levels gradually decreased with time after administration of CDDP solution, whereas they increased with time after CDDP-SLX-LIP administration. Furthermore, administration of S-LIP resulted in approximately three times higher accumulation in tumors than L-LIP at 24 and $48 \mathrm{~h}$.

\section{Discussion}

Liposomes binding SLX on the cell surface have been reported to be useful tools for the tumor-selective delivery of antitumor drugs. Liposomes with SLX are as negatively charged as vascular endothelial cells, erythrocytes and leukocytes, meaning that they repell each other electrically. Moreover, the hydrophilic surface of the liposomes prevented opsonin protein adsorption in plasma and phagocytosis by macrophages. Therefore, retention of liposomes in blood was extended. SLX-liposomes containing fluorescent Cy5.5 (SLX-Lipo-Cy5.5) accumulated in areas of inflammation or tumor regions after intravenous administration. In addition, the accumulation of SLX-LipoCy5.5 shifted from blood vessels to the surrounding tissues (16). Tsuruta et al. (20) reported that Dox-Lipo-SLX, an SLX-liposome containing doxorubicin, had an affinity for Eselectin and efficiently delivered doxorubicin to cells expressing E-selectin. Additionally, the different outcomes obtained between Dox-Lipo-SLX and Dox-Lipo might have 

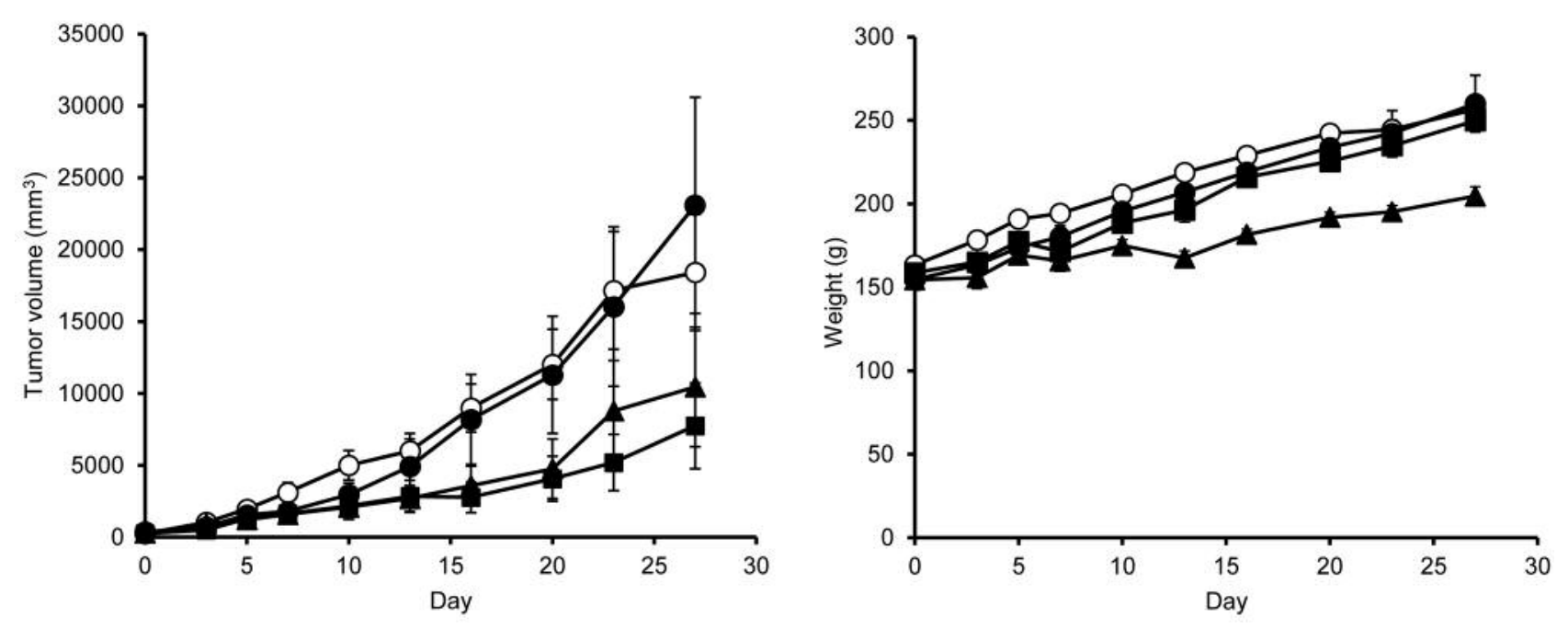

Figure 2. Effects of L-LIP in SD rats bearing MRMT-1 tumors. Drugs were administered intravenously via the tail vein at day 0, 5 and 10. Open circle: No-treatment; closed circle: empty liposome; closed triangle: CDDP solution (4 mg CDDP/kg); closed square: L-LIP (8 mg CDDP/kg). Data represent the mean \pm SEM $(n=5-7)$.
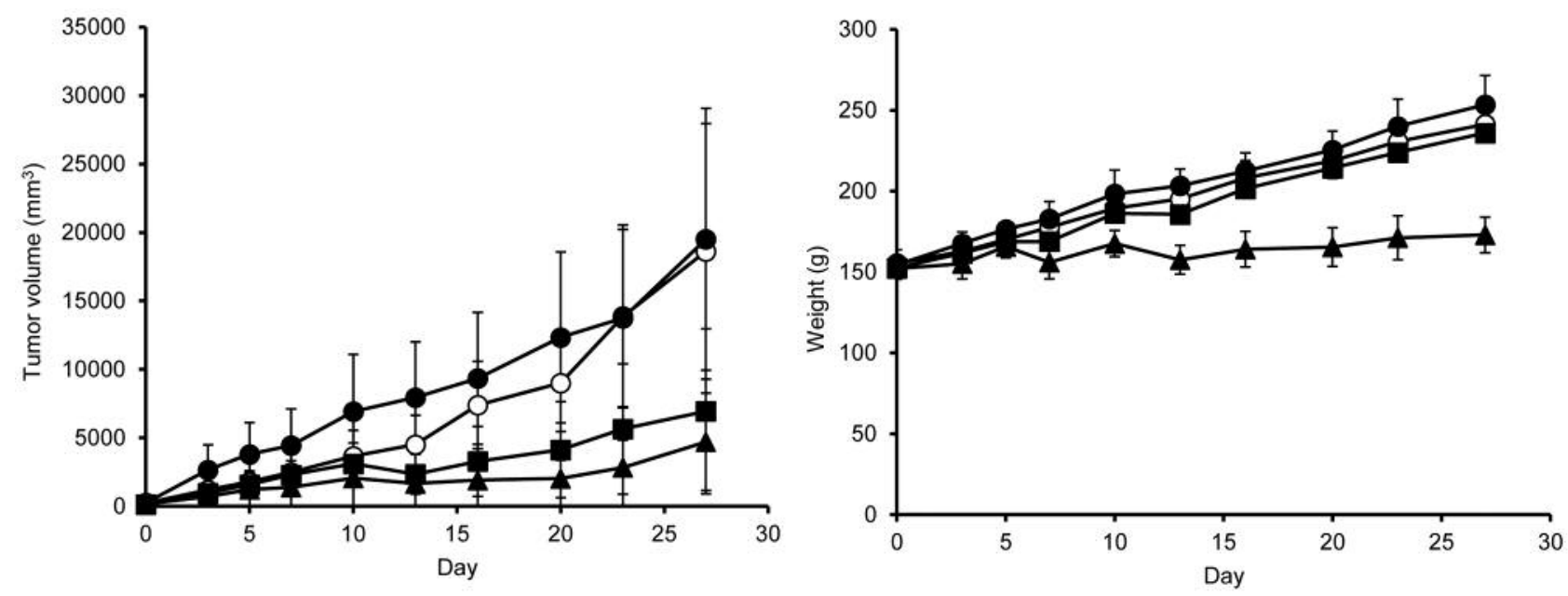

Figure 3. Effects of S-LIP in SD rats bearing MRMT-1 tumors. Drugs were administered intravenously via the tail vein at day 0, 5 and 10. Open circle: No-treatment; closed circle: empty liposome; closed triangle: CDDP solution (4 mg CDDP/kg); closed square: S-LIP (8 mg CDDP/kg). Data represent the mean $\pm \operatorname{SEM}(n=5)$.

been a consequence of active versus passive targeting. These findings suggested that SLX-liposomes may be useful carriers of antitumor drugs and be the basis for active targeting drug delivery systems.

A method to efficiently deliver CDDP directly to the tumor is needed to improve the therapeutic response while potentially avoiding significant side effects. For platinum derivatives, liposomal formulations (SPI-077, Lipoplatin, SpHL, and Lipoxal) in preclinical and clinical trials have shown an ability to reduce side effects while maintaining efficacy. All of them contained DSPE-PEG2000 in their lipid bilayer to prolong blood circulation and allow its passive accumulation in tumors. It was clear from a comparison between SPI-077 and Lipoplatin that the ratio of the incorporated CDDP to lipid weight of liposomal formulations is important for sufficient delivery of CDDP to the tumor because CDDP is water insoluble and has low lipophilicity $(6,7)$. Therefore, an SLX-liposome that enables 

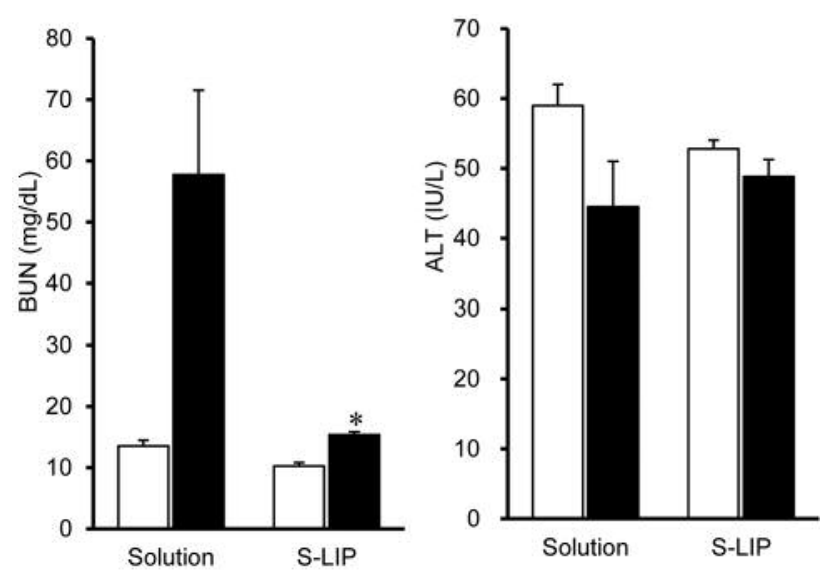

Figure 4. Effects of S-LIP on kidney and liver of SD rats. Drugs were administered intravenously via the tail vein. Doses of CDDP solution and S-LIP were $4 \mathrm{mg} C D D P / \mathrm{kg}$ and $8 \mathrm{mg} / \mathrm{kg}$, respectively. Open column: Before administration; closed column: 96 h after administration. Data represent the mean \pm SEM $(n=5)$. * $p<0.05$ vs. $C D D P$ solution at the same time point.

active targeting to tumor tissues can be expected to have a higher antitumor effect than PEGylated liposomal formulations by having high CDDP content.

The original CDDP-SLX-LIP formulation, which was defined as L-LIP, had a high drug-to-lipid ratio of 1:14, which was equivalent to that of Lipoplatin with a ratio of 1:10. The particle size of L-LIP was $182 \mathrm{~nm}$, which was larger than those of SPI-077 and Lipoplatin at approximately $110 \mathrm{~nm}$. Because particle size affects accumulation in tumor tissues, the smaller CDDP-SLX-LIP formulation, which was defined as S-LIP, was expected to show increased tumor accumulation. The particle size of S-LIP was $131 \mathrm{~nm}$ and $\mathrm{S}$-LIP required a higher drug-to-lipid ratio (1:30) to hold $2 \mathrm{mg} / \mathrm{mL}$ of CDDP than L-LIP because S-LIP had a lower entrapment efficiency caused by smaller internal spaces compared with those of L-LIP. While there was no difference in the lipid composition between L-LIP and S-LIP, there was a clear difference in the release profile. Both liposomes exhibited an initial release, which was observed immediately after the start of the incubation of liposomes in saline, and a sustained release following the initial release. The release from L-LIP was 4.7-times higher in the initial phase and 1.8times higher in the sustained phase than that from S-LIP. This indicated that a higher number of lipid molecules in the liposome per CDDP molecule resulted in a slower release of CDDP from the liposome. The initial phase of the release from CDDP-SLX-LIP was unexpected and might have been due to bursting of liposomes or a rapid diffusion from liposomes. However, it would be difficult to reproduce the bursting of liposomes. The initial and sustained release phases might have been due to a two-stage diffusion process. This would mean that there were two fractions with CDDP present in CDDP-SLX-LIP, which might have been caused by the indirect CDDP encapsulation using the conversion from cis-diamminedinitratoplatin. The detailed mechanism is unknown and necessary to elucidate in the future.

PEG-modified nanocarriers allow for a long retention in the blood by avoiding entrapment in the RES by the EPR effect. It has been reported that even in nanocarriers encapsulating CDDP, retention in the blood is considerably long and accumulation in the liver and spleen increases with time $(10,21)$. Compared with to nanocarriers, the retention of CDDP-SLX-LIP in blood was somewhat shorter and accumulation in liver and spleen were substantially higher immediately after administration. However, tumor accumulation was initially low, but increased 24- and 48-h after administration. Hirai et al. (16) reported that SLX-LipoCy5.5 accumulated significantly in areas of inflammation and the tumors. In addition, accumulation increased gradually until $48 \mathrm{~h}$ because SLX-Lipo-Cy5.5 in the blood stream was recognized and trapped by E-selectin expressed on blood vessels. Therefore, the tumor-selective accumulation of CDDP-SLX-LIP was a result of the affinity of the SLX moiety to the E-selectin in the blood vessels of tumor tissues.

The two CDDP-SLX-LIPs showed excellent inhibition of the growth of tumors without obvious toxicity. The liposomes required two-times higher dose than that of CDDP solution to provide a similar antitumor effect, but CDDP solution caused the death of rats when treated at the same dose as that of CDDP-SLX-LIP. At doses showing comparable antitumor effects, treatment with CDDP solution caused significant weight loss and elevated BUN levels, but treatment with CDDP-SLX-LIPs caused no obvious change in either. The low toxicity of CDDP-SLX-LIP was based on the slow release of CDDP from liposomes as described above. In addition, the tumor-selective effects of CDDP-SLX-LIP resulted from high tumor accumulation and the slow release characteristic of the liposomes. Interestingly, there was almost no difference in the antitumor effects of L-LIP and S-LIP. Treatment with L-LIP resulted in less accumulation in the tumor tissues and a higher release of CDDP compared with S-LIP. In other words, treatment with S-LIP resulted in higher tumor accumulation, but did not have stronger antitumor effects because of its slower release characteristics compared with those of L-LIP. The platinum amounts measured in the tissues were the sum of encapsulated and released platinum compounds. Like SPI-077, CDDP-SLX-LIP also must release CDDP from the liposome to demonstrate antitumor effects. In fact, the slow release characteristic of SPI-077 was reported to interfere with its in vivo antitumor efficacy and cause the clinical trial to fail (22). In contrast, SPI-077 B-103, which was developed to enhance the release of CDDP from the liposomes, had lower 


\section{Plasma}
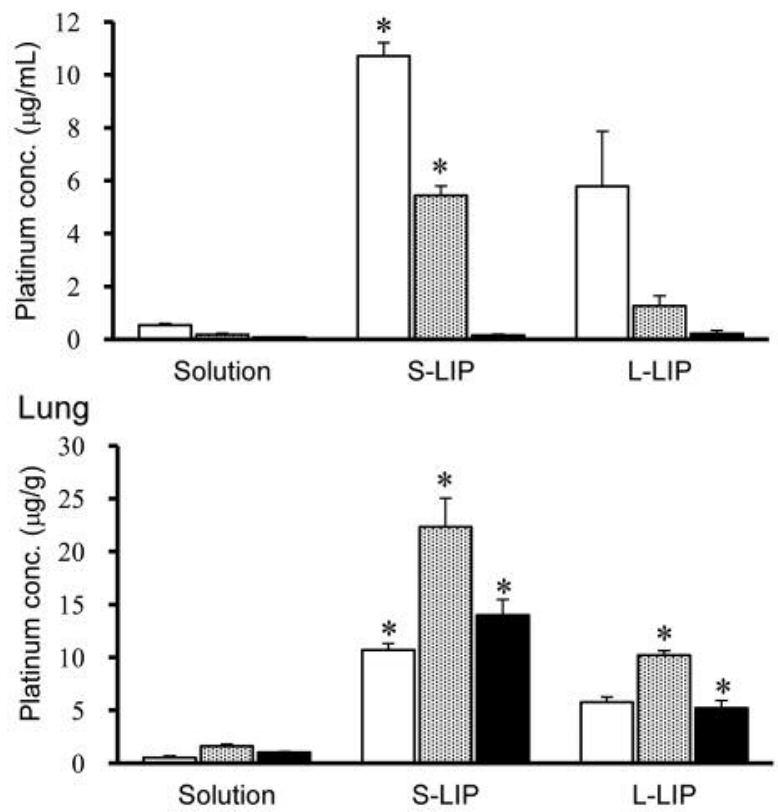

Liver

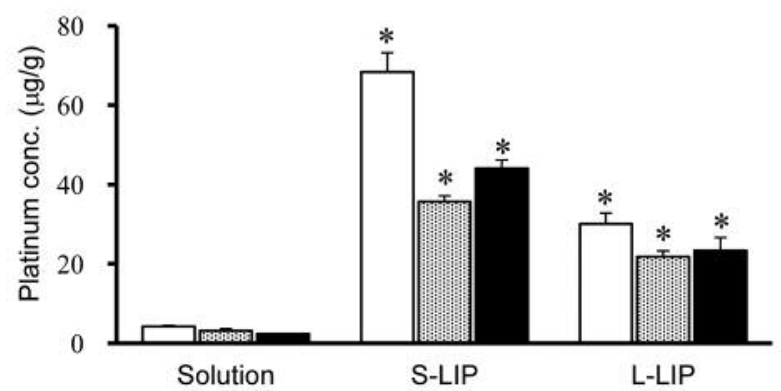

Spleen

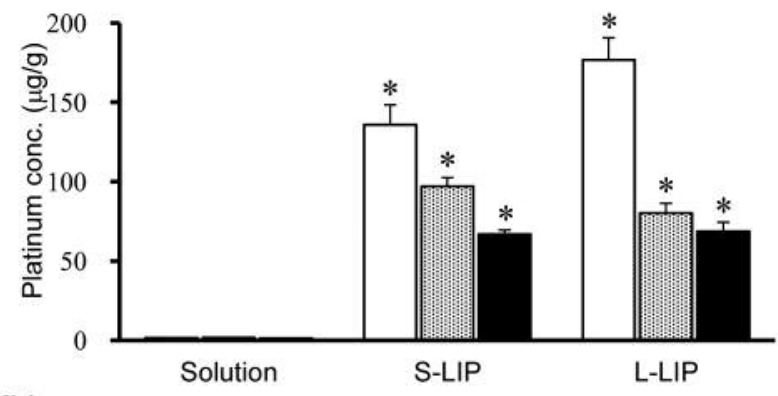

Kidney

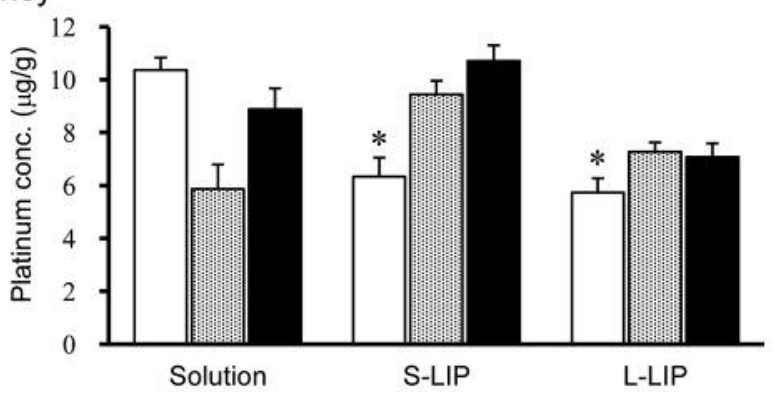

Tumor

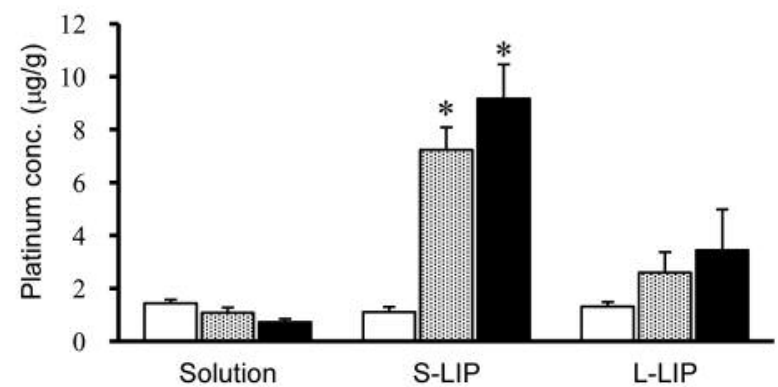

Figure 5. Biodistribution of CDDP-SLX-LIP in SD rats bearing MRMT-1 tumors. Drugs were administered intravenously via the tail vein. Doses of $C D D P$ solution and CDDP-SLX-LIP were $4 \mathrm{mg} / \mathrm{kg}$ and $8 \mathrm{mg} / \mathrm{kg}$, respectively. Open column: $1 \mathrm{~h}$ after administration; dotted column: $24 \mathrm{~h}$ after administration; closed column: 48 h after administration. Data represent the mean $\pm S E M(n=5) . * p<0.05$ vs CDDP solution at the same time point.

tumor accumulation and required dose reduction owing to the onset of toxicity (11). Zhao et al. (23) compared three different liposomes that contained dimyristoyl phosphatidylcholine (DMPC), DPPC, or distearoyl phosphatidylcholine (DSPC) as the main lipid component loaded with aquated CDDP to improve the delivery of aquated CDDP. They found that the DSPC liposomal formulation with an increased transition temperature exhibited a lower drug release rate, prolonged pharmacokinetics, improved tumor delivery, and enhanced antitumor efficacy. The DSPC formulation exhibited an extremely slow sustained release with a release rate of approximately $2 \%$ in $72 \mathrm{~h}$, but a very high tumor accumulation of about $20 \%$ injection dose/g in $24 \mathrm{~h}$. Therefore, to obtain strong antitumor activity, a balance between tumor accumulation and release rate is necessary for the liposome formulation. That is, in the case of highly sustained-release formulations, a sufficient antitumor effect cannot be obtained unless high tumor accumulation is achieved. A formulation that combines extremely high sustained release and tumor accumulation can exert tumor-selective antitumor effects.

In this experiment, two CDDP-SLX-LIPs of different sizes displayed a two-step release profile with differences in release rates. At doses at which CDDP solution exerted a sufficient antitumor effect, weight gain suppression, hair loss, and increased BUN values were observed. Conversely, treatment with CDDP-SLX-LIP resulted in equivalent antitumor effects without the adverse effects. In addition, the antitumor effects were the same regardless the size of the 
liposomes. Treatment with CDDP-SLX-LIPs also resulted in a long retention in the blood and high tumor accumulation; both these parameters were affected by liposome size. Control of liposome size and drug release rate is presumed to be a crucial factor that leads to enhancement of the antitumor effect of CDDP-SLX-LIP.

\section{References}

1 Dilruba S and Kalayda GV: Platinum-based drugs: past, present and future. Cancer Chemother Pharmacol 77: 1103-1124, 2016.

2 Gabizon A and Martin F: Polyethylene glycol-coated (pegylated) liposomal doxorubicin. Rationale for use in solid tumours. Drugs 54: 15-21, 1997.

3 Ogawara K, Un K, Tanaka K, Higaki $\mathrm{K}$ and Kimura T: In vivo anti-tumor effect of PEG liposomal doxorubicin (DOX) in DOXresistant tumor-bearing mice: Involvement of cytotoxic effect on vascular endothelial cells. J Control Release 133: 4-10, 2009.

4 Maruyama K: Intracellular targeting delivery of liposomal drugs to solid tumors based on EPR effects. Adv Drug Deliv Rev 63: 161-169, 2011.

5 Suk JS, Xu Q, Kim N, Hanes J and Ensign LM: PEGylation as a strategy for improving nanoparticle-based drug and gene delivery. Adv Drug Deliv Rev 99: 28-51, 2016.

6 Zalba S and Garrido MJ: Liposomes, a promising strategy for clinical application of platinum derivatives. Expert Opin Drug Deliv 10: 829-844, 2013.

7 Liu D, He C, Wang AZ and Lin W: Application of liposomal technologies for delivery of platinum analogs in oncology. Int $\mathrm{J}$ Nanomedicine 8: 3309-3319, 2013.

8 Oberoi HS, Nukolova NV, Kabanov AV and Bronich TK: Nanocarriers for delivery of platinum anticancer drugs. Adv Drug Deliv Rev 65: 1667-1685, 2013.

9 Duan X, He C, Kron SJ and Lin W: Nanoparticle formulations of cisplatin for cancer therapy. Wiley Interdiscip Rev Nanomed Nanobiotechnol 8: 776-891, 2016.

10 Newman MS, Colbern GT, Working PK, Engbers C and Amantea MA: Comparative pharmacokinetics, tissue distribution, and therapeutic effectiveness of cisplatin encapsulated in longcirculating, pegylated liposomes (SPI-077) in tumor-bearing mice. Cancer Chemother Pharmacol 43: 1-7, 1999.

11 Zamboni WC, Gervais AC, Egorin MJ, Schellens JH, Zuhowski EG, Pluim D, Joseph E, Hamburger DR, Working PK, Colbern G, Tonda ME, Potter DM and Eiseman JL: Systemic and tumor disposition of platinum after administration of cisplatin or STEALTH liposomal-cisplatin formulations (SPI-077 and SPI077 B103) in a preclinical tumor model of melanoma. Cancer Chemother Pharmacol 53: 329-336, 2004.

12 Devarajan P, Tarabishi R, Mishra J, Ma Q, Kourvetaris A, Vougiouka M and Boulikas T: Low renal toxicity of lipoplatin compared to cisplatin in animals. Anticancer Res 24: 2193-2200, 2004.
13 Boulikas T: Molecular mechanisms of cisplatin and its liposomally encapsulated form, Lipoplatin. Lipoplatin as a chemotherapy and antiangiogenesis drug. Cancer Therapy 5: 351-376, 2007.

14 Uchino H, Matsumura Y, Negishi T, Koizumi F, Hayashi T, Honda T, Nishiyama N, Kataoka K, Naito S and Kakizoe T: Cisplatin-incorporating polymeric micelles (NC-6004) can reduce nephrotoxicity and neurotoxicity of cisplatin in rats. $\mathrm{Br}$ J Cancer 93: 678-687, 2005.

15 Matsumura Y: Poly (amino acid) micelle nanocarriers in preclinical and clinical studies. Adv Drug Deliv Rev 60: 899-914, 2008.

16 Hirai M, Minematsu H, Kondo N, Oie K, Igarashi K and Yamazaki N: Accumulation of liposome with Sialyl Lewis X to inflammation and tumor region: application to in vivo bioimaging. Biochem Biophys Res Commun 353: 553-558, 2007.

17 Hashida N, Ohguro N, Yamazaki N, Arakawa Y, Oiki E, Mashimo $\mathrm{H}$, Kurokawa $\mathrm{N}$ and Tano $\mathrm{Y}$ : High-efficacy sitedirected drug delivery system using sialyl-Lewis $\mathrm{X}$ conjugated liposome. Exp Eye Res 86: 138-149, 2007.

18 Hirai M, Hiramatsu Y, Iwashita S, Otani T, Chen L, Li YG, Okada M, Oie K, Igarashi K, Wakita H and Seno M: E-selectin targeting to visualize tumors in vivo. Contrast Media Mol Imaging 5: 70-77, 2010.

19 Hirai M, Minematsu H, Hiramatsu Y, Kitagawa H, Otani T, Iwashita S, Kudoh T, Chen L, Li Y, Okada M, Salomon DS, Igarashi K, Chikuma M and Seno M: Novel and simple loading procedure of cisplatin into liposomes and targeting tumor endothelial cells. Int J Pharm 391: 274-283, 2010.

20 Tsuruta W, Tsurushima H, Yamamoto T, Suzuki K, Yamazaki N and Matsumura A: Application of liposomes incorporating doxorubicin with sialyl Lewis $\mathrm{X}$ to prevent stenosis after rat carotid artery injury. Biomaterials 30: 118-125, 2009.

21 Bandak S, Goren D, Horowitz A, Tzemach D and Gabizon A: Pharmacological studies of cisplatin encapsulated in longcirculating liposomes in mouse tumor models. Anticancer Drugs 10: 911-920, 1999.

22 Harrington KJ, Lewanski CR, Northcote AD, Whittaker J, Wellbank H, Vile RG, Peters AM and Stewart JS: Phase I-II study of pegylated liposomal cisplatin (SPI-077) in patients with inoperable head and neck cancer. Ann Oncol 12: 493-496, 2001.

23 Zhao Y, May JP, Chen IW, Undzys E and Li SD: A study of liposomal formulations to improve the delivery of aquated cisplatin to a multidrug resistant tumor. Pharm Res 32: 3261-3268, 2015.

Received June 7, 2017

Revised June 28, 2017

Accepted June 30, 2017 